\title{
Article \\ Effects of Mixing Volatile Fatty Acids as Carbon Sources on Rhodospirillum rubrum Carbon Metabolism and Redox Balance Mechanisms
}

\author{
Paloma Cabecas Segura ${ }^{1}$ (D), Quentin De Meur ${ }^{1}$, Audrey Tanghe ${ }^{2}$, Rob Onderwater ${ }^{2}$, Laurent Dewasme ${ }^{3} \mathbb{D}$, \\ Ruddy Wattiez ${ }^{1}$ and Baptiste Leroy ${ }^{1, *}$
}

1 Laboratory of Proteomics and Microbiology, University of Mons, 7000 Mons, Belgium; paloma.cabecassegura@umons.ac.be (P.C.S.); q.demeur@gmail.com (Q.D.M.); ruddy.wattiez@umons.ac.be (R.W.)

2 Materia Nova ASBL, Parc Initialis, Avenue Copernic 3, 7000 Mons, Belgium; Audrey.Tanghe@MATERIANOVA.BE (A.T.); Rob.Onderwater@MATERIANOVA.BE (R.O.)

3 Systems, Estimation, Control and Optimization Group, University of Mons, 7000 Mons, Belgium; laurent.dewasme@umons.ac.be

* Correspondence: baptiste.leroy@umons.ac.be; Tel.: +32-65-372271

check for updates

Citation: Cabecas Segura, P.; De Meur, Q.; Tanghe, A.; Onderwater, R.; Dewasme, L.; Wattiez, R.; Leroy, B. Effects of Mixing Volatile Fatty Acids as Carbon Sources on Rhodospirillum rubrum Carbon Metabolism and Redox Balance Mechanisms.

Microorganisms 2021, 9, 1996. https:// doi.org/10.3390/microorganisms9091996

Academic Editor: Hitoshi Miyasaka

Received: 10 August 2021

Accepted: 16 September 2021

Published: 21 September 2021

Publisher's Note: MDPI stays neutral with regard to jurisdictional claims in published maps and institutional affiliations.

Copyright: (c) 2021 by the authors. Licensee MDPI, Basel, Switzerland. This article is an open access article distributed under the terms and conditions of the Creative Commons Attribution (CC BY) license (https:/ / creativecommons.org/licenses/by/ $4.0 /)$.

\begin{abstract}
Rhodospirillum rubrum has a versatile metabolism, and as such can assimilate a broad range of carbon sources, including volatile fatty acids. These carbon sources are gaining increasing interest for biotechnological processes, since they reduce the production costs for numerous valueadded compounds and contribute to the development of a more circular economy. Usually, studies characterizing carbon metabolism are performed by supplying a single carbon source; however, in both environmental and engineered conditions, cells would rather grow on mixtures of volatile fatty acids (VFAs) generated via anaerobic fermentation. In this study, we show that the use of a mixture of VFAs as carbon source appears to have a synergy effect on growth phenotype. In addition, while propionate and butyrate assimilation in Rs. rubrum is known to require an excess of bicarbonate in the culture medium, mixing them reduces the requirement for bicarbonate supplementation. The fixation of $\mathrm{CO}_{2}$ is one of the main electron sinks in purple bacteria; therefore, this observation suggests an adaptation of both metabolic pathways used for the assimilation of these VFAs and redox homeostasis mechanism. Based on proteomic data, modification of the propionate assimilation pathway seems to occur with a switch from a methylmalonyl-CoA intermediate to the methylcitrate cycle. Moreover, it seems that the presence of a mixture of VFAs switches electron sinking from $\mathrm{CO}_{2}$ fixation to $\mathrm{H}_{2}$ and isoleucine production.
\end{abstract}

Keywords: purple bacteria; VFA; Rs. rubrum; dihydrogen; proteomic; redox homeostasis; photoheterotrophy

\section{Introduction}

Rhodospirillum rubrum (Rs. rubrum), a purple non-sulfur bacteria (PNSB) belonging to the alpha-proteobacteria class, has been extensively studied for its versatile metabolism. This bacterium can assimilate a broad range of substrates, ranging from sugar to volatile fatty acids [1]. VFAs very often result from waste stream treatment through fermentation [2], making them particularly interesting carbon sources for biotechnological processes [3-7]. The valorization of this type of carbon sources to produce high added value compounds such as pigments and biopolymers [2,8] using purple bacteria could help increase the profitability of the biobased industry and lead towards a circular economy. In waste streams, VFA $_{S}$ are always found in mixtures. Even though the compositions and proportions of VFA $_{S}$ depend on many factors, the most abundant VFAs produced are usually acetate, butyrate, and propionate [9-14]. The metabolic process involved in the assimilation of these VFA $_{S}$ has been studied in PNSB. In Rs. rubrum, for example, the net carbon assimilation of acetate occurs via the use of an alternative anaplerotic pathway, the ethylmalonyl-CoA 
pathway (EMC) [15-17]. When propionate is used as a carbon source, propionyl-CoA is carboxylated in methylmalonyl-CoA before being converted to succinate, which enters the tricarboxylic acid cycle (TCA) [18]. Concerning butyrate assimilation, a recent study carried out on Rs. rubrum highlighted the use of the EMC pathway for butyrate assimilation, as well as the newly proposed methylbutanoyl-CoA pathway (MBC) [19].

The previous studies were performed on culture supplemented with only one carbon source, meaning they did not properly reflect the events that occur under more environmental or engineered conditions, where several VFAs would be simultaneously present. Some studies carried out on cultures of PNSB growing on a mixture of VFAs already highlighted the synergic effect on the uptake rate of VFA and the sequential uptake of the various VFAs simultaneously present in the medium [4,5]; however, the mechanisms involved in these phenomena have not yet been described. We recently showed that in presence of a mixture of VFAs, a lesser requirement for bicarbonate supplementation was observed in Rs. rubrum [20]. We also observed that propionate and acetate inhibited butyrate assimilation in binary mixtures [20]. In addition, we showed that when Rs. rubrum was cultivated using a mixture of propionate and butyrate, contrary to what is observed when these VFAs are provided as sole carbon sources, bicarbonate supplementation was not required anymore. The bicarbonate supplementation is supposedly required to maintain the redox balance of the cell, with the fixation of $\mathrm{CO}_{2}$ being one of the main electron sinks in PSNB. This result suggests that alternative redox homeostasis mechanisms must be involved when a mixture of VFA ${ }_{S}$ is used as a carbon source. The present study aims, therefore, to unravel the way propionate affects butyrate assimilation, as well as the molecular aspects of propionate and butyrate assimilation when supplied as unique organic carbon sources or as a mixture in Rs. rubrum.

\section{Materials and Methods}

\subsection{Bacterial Strain, Culture Medium, and Growth Conditions}

The strains used was Rhodospirillum rubrum S1H (ATCC15903). The growth basal medium used for photoheterotrophic culture conditions of Rs. rubrum was the basal salt medium of Segers and Verstraete described by Suhaimi [21,22]. This basal medium was supplemented with $\mathrm{NH}_{4} \mathrm{CI}(35 \mathrm{mM})$ as the nitrogen source, $\mathrm{NaHCO}_{3}(3 \mathrm{mM}$ or $50 \mathrm{mM})$, and biotin $(0.06 \mathrm{mM})$. Different VFAs were used as carbon sources, including a mixture of propionate $(20.6 \mathrm{mM})$ and butyrate $(15.5 \mathrm{mM})$, propionate $(41.66 \mathrm{mM})$, or butyrate $(31.25 \mathrm{mM})$. The $\mathrm{pH}$ was adjusted to 6.9. Rs. rubrum was grown under anaerobic phototrophic conditions in $50 \mathrm{~mL}$ sealed serum flasks under $50 \mu \mathrm{mole} \cdot \mathrm{m}^{-2} \cdot \mathrm{sec}^{-1}$ of light intensity supplied by halogen lamps (Sencys, Amsterdam, The Netherlands; $10 \mathrm{~W}$; 100 lumens; $2650 \mathrm{~K}$ ) at $30^{\circ} \mathrm{C}$ with a rotary shaking at $200 \mathrm{rpm}$. Each culture condition was achieved with three biological replicates, except for the cultivation experiments with a mixture of acetate and excess butyrate, which were carried out on four biological replicates. Nitrogen gas was used to purge oxygen from the upper gas phase and the flasks were hermetically sealed. The cultures were inoculated at a starting $\mathrm{OD}_{680 \mathrm{~nm}}$ range of $0.4-0.5$ and the growth was monitored following the turbidity at $\mathrm{OD}_{680 \mathrm{~nm}}$.

\subsection{Volatile Fatty Acid Consumption Monitoring by HPLC}

The monitoring of VFA consumption by Rs. rubrum was performed on culture supernatants obtained by centrifugation at $16,000 \mathrm{~g}$ for $10 \mathrm{~min}$ at $4{ }^{\circ} \mathrm{C}$ and stored at $-20^{\circ} \mathrm{C}$ before analysis. Aliquots $(100 \mu \mathrm{L})$ of the culture supernatant were analyzed by HPLC refractometry (Waters 2695 separation Module; Waters 2414 Refractive Index Detector, Waters, Milford, MA, USA). The separation was performed in isocratic mode using a Shodex Sugar SH1011 column ( $300 \mathrm{~mm} \times 8 \mathrm{~mm}$, SHODEX, New York, NY, USA) with $5 \mathrm{mM} \mathrm{H}_{2} \mathrm{SO}_{4}$ as the mobile phase. The detection was performed using refractometry at $210 \mathrm{~nm}$. 


\subsection{Polyhydroxyalkanoates Quantification}

The method used to quantify polyhydroxyalkanoates content was adapted from a previously described method [23]. To determine the PHA composition and content, $500 \mu \mathrm{L}$ of culture was centrifuged at $8000 \mathrm{rpm}$ for $5 \mathrm{~min}$. The pellet was resuspended with $500 \mu \mathrm{L}$ of chloroform and added to a $10 \mathrm{~mL}$ screw cap glass tubes with $2 \mathrm{ml}$ of a solution containing $1.94 \mathrm{~mL}$ of methanol, $0.06 \mathrm{~mL}$ of sulfuric acid, and $0.2 \mathrm{mg}$ of toluic acid. The tubes were then placed in a thermostatically regulated bath at $100^{\circ} \mathrm{C}$ for $210 \mathrm{~min}$. After the reaction, $1 \mathrm{~mL}$ of distilled water was added and the tube was shaken vigorously. After phase separation, the organic phase (bottom layer) was removed and transferred to a small screw cap glass vial. The methyl esters were analyzed using gas chromatography using a SHIMADZU GC-MS QP2010S instrument (Shimadzu, Kyoto, Japan) equipped with an Optima ${ }^{\circledR} 5$ capillary column ( $30 \mathrm{~m} / 0.25 \mathrm{~nm}$; Macherey-Nagels, Düren, Germany) and a flame ionization detector. Afterwards, $2 \mu \mathrm{L}$ of the organic phase was analyzed after spitless injection. Helium $(20.2 \mathrm{~mL} / \mathrm{min})$ was used as the carrier gas. The temperature of the injector was $250^{\circ} \mathrm{C}$.

\subsection{Proteomic Analysis}

Quantitative proteomic analysis was performed on protein extracts from Rs. rubrum $\mathrm{S} 1 \mathrm{H}$ strain cultivated in butyrate, propionate, and a mix of propionate and butyrate containing medium with an excess of carbonate. Cells from 5 biological replicates were harvested by centrifugation $\left(16,000 \times g, 10 \mathrm{~min}\right.$ at $\left.4{ }^{\circ} \mathrm{C}\right)$ when the $\mathrm{OD}_{680 \mathrm{~nm}}$ reached 2.7. Proteins were extracted in a guanidine $\mathrm{HCl} 6 \mathrm{M}, \mathrm{K}_{2} \mathrm{HPO}_{4} 50 \mathrm{mM}$ buffer by sonication $(3 \times 10 \mathrm{~s}$, amplitude $20 \%$, IKA U50 sonicator). The protein concentration was determined using the Bradford method, with bovine gamma-globulin used as the standard [24]. Aliquots of extracted proteins $(100 \mu \mathrm{g})$ were reduced using DTE, alkylated with iodoacetamide, and precipitated with acetone. The proteolytic peptides were obtained by overnight enzymatic digestion at $37^{\circ} \mathrm{C}$ using trypsin at a ratio of 2:50 (w/w). Digestion was stopped with $0.1 \%$ formic acid ( $v / v$, final concentration). The suspension was purified using Pierce ${ }^{\mathrm{TM}}$ C18 spin tips. The final amount of peptide was quantified using Pierce ${ }^{\mathrm{TM}}$ quantitative peptide. Proteins were identified and quantified following a label-free UHPLC-HRMS/MS platform (Eksigent Ekspert nano LC400 and Triple TOFTM 6600 AB Sciex, Singapore) in SWATH mode of acquisition. Peptides $(2 \mu \mathrm{g})$ were separated on a C18 column $(3 \mu \mathrm{m}$, $75 \mu \mathrm{m} \times 15 \mathrm{~cm}$, Dionex, Sunyvale, CA, USA) using a linear acetonitrile (ACN) gradient (2 to $35 \%$ of acetonitrile $(v / v), 120 \mathrm{~min}$ ) in water containing $0.1 \%(v / v)$ formic acid, at a flow rate of $300 \mathrm{~nL} / \mathrm{min}^{-1}$. To achieve the greatest possible retention time stability, the column was equilibrated with $10 \mathrm{vol} 5 \%$ acetonitrile $(\mathrm{ACN})$ before each injection. Mass spectra were acquired over the range of 400-1250 m/z in high-resolution mode (resolution > 35,000), with a $50 \mathrm{~ms}$ accumulation time. SWATH MS/MS spectra were acquired in data-independent acquisition (DIA) mode over the same $\mathrm{m} / \mathrm{z}$ range with 50 variable SWATH windows. SWATH data were used to identify peptides via comparison with a reference spectral library obtained using a data-dependent acquisition (DDA) experiment on protein samples extracted from Rs. rubrum $\mathrm{S} 1 \mathrm{H}$ grown on different carbon sources (succinate, acetate, propionate, butyrate, valerate, hexanoate) and with different light intensity levels $\left(50 \mu \mathrm{molm}^{-2} \mathrm{~s}^{-1}\right.$, $150 \mu \mathrm{molm}^{-2} \mathrm{~s}^{-1}$ ). The sample preparation and separation procedures used to construct the library were identical to those mentioned above. For library creation, the instrument was operated in data-independent acquisition mode and MS/MS spectra were acquired over the range of 100-1800 m/z. The precursor selection parameters were an intensity threshold of 200 c.p.s and accumulation time of $50 \mathrm{~ms}$. Spectra were identified via comparison with the Rs. rubrum ATCC11170 UniProt entries (UPID/UP000001929/May 2013) using the AB Sciex ProteinPilot ${ }^{\mathrm{TM}} 5.0$ software. For peptide quantification, AB Sciex PeakView ${ }^{\mathrm{TM}}$ 2.2 software and SWATH ${ }^{\mathrm{TM}}$ processing were used to calculate XICs for the six highest fragments for the top five peptides from all identified proteins at a false discovery rate below $1 \%$. Only unmodified and unshared peptides were subject to quantification. Peptides were also excluded when the confidence in their identification was below 0.99. A mass tolerance 
of $0.015 \mathrm{~m} / \mathrm{z}$ and a retention time window of $2 \mathrm{~min}$ were also applied for XIC creation and integration. The areas under the curve of the XIC were exported to MarkerView TM 1.3. software for normalization based on the summed areas of the entire run and statistical analyses. Only proteins with fold changes, greater or lower than 1.5 or $0.66, p$-values under 0.05 and quantified with at least two peptides were further considered.

\subsection{Isoleucine Quantification}

Branched amino acids (BCAAs) were extracted from pellets issued from the centrifugation of $500 \mu \mathrm{L}$ cultures. The pellets were resuspended in $1.5 \mathrm{~mL}$ of methanol/chloroform solution $(1: 2, v: v)$. The resuspended pellets then underwent five freeze-thawing cycles, then $400 \mu \mathrm{L}$ of MilliQ water was added and the mixture was centrifuged (5000 rpm, $10 \mathrm{~min}, 4^{\circ} \mathrm{C}$ ). The upper aqueous phase was recovered and submitted to SpeedVac before being stored at $-20{ }^{\circ} \mathrm{C}$ until analysis. The obtained pellet was then resuspended in mass spectrometer loading solvent consisting of $0.2 \%$ formic acid in ultrapure MS-grade water. The BCAA content was analyzed using an Eksigent LC425 system coupled to a Q-TRAP instrument (AB Sciex Q-Trap-6500+; ABSciex, Singapore) used in multiple reaction monitoring (MRM) modes. Peptides were separated on a C18 YMC-Triat $0.3 \times 150 \mathrm{~mm}$ column operated at a flow rate of $5 \mu \mathrm{L} / \mathrm{min}$ in isocratic mode (3\% acetonitrile $(v / v)$, formic acid $0.1 \%(v / v)$ ). The following transitions were used to quantify the following amino acids: arginine 175/116 and isoleucine 132/69. In order to avoid extraction bias, isoleucine abundance was expressed as the ratio of the area under the curve of the isoleucine transition to the area under the curve of the specific transition of the arginine.

\section{6. $\mathrm{H}_{2}$ Quantification}

$\mathrm{H}_{2}$ was sampled from the culture headspace using a gas-tight syringe and was quantified using a Trace ${ }^{\text {TM }} 1300$ gas chromatograph (Thermofisher, Waltham, MA, USA) equipped with a thermal conductivity detector and a HayeSep N Sulfinert column $(0.25 \mathrm{~m}$ by $1 \mathrm{nmm}$; Agilent, Santa Clara, CA, USA). Argon was used as the carrier gas at a flow rate of $1 \mathrm{~mL} / \mathrm{min}$. The oven temperature was $60{ }^{\circ} \mathrm{C}$, the inlet temperature was $200{ }^{\circ} \mathrm{C}$, and the detector temperature was $110^{\circ} \mathrm{C}$.

\subsection{Acetolactate Synthase Specific Activity}

Cells were harvested after 24 or $72 \mathrm{~h}$ of culture before being centrifuged and washed using phosphate buffer $(50 \mathrm{mM}, \mathrm{pH}=7.0)$. Cells were lysed in $100 \mu \mathrm{L}$ phosphate buffer using $25 \mathrm{mg}$ of glass beads and lysozyme ( $1 \mathrm{mg} / \mathrm{mL}$ final concentration), then the protein concentration was determined using the Bradford method [24], with bovine gamma globulin used as the standard. Acetolactate synthase activity was tested as described previously [25]. Acetolactate produced after one hour was assayed at a single endpoint by conversion to acetoin, which was detected using the reaction method described by Westerfeldt [26] and quantified through the use of a standard curve after subtraction of the acetoin produced without substrate. The acetoin content was then normalized based on the protein content.

\section{Results}

Based on the recently observed inhibition of acetate and propionate via the assimilation of butyrate in purple bacteria [20], we decided to study the molecular mechanism of propionate and butyrate assimilation by Rs. rubrum when supplied as a mixture. The VFA uptake profile showed that propionate induces a delay in the assimilation of butyrate, as previously observed in Rs. rubrum (Figure 1) [20]. The growth curve can consequently be divided into a propionate or a butyrate assimilation phase, as shown in Figure 1c. 


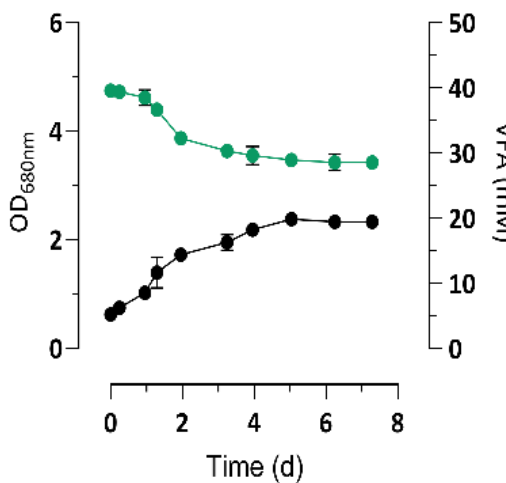

(a)

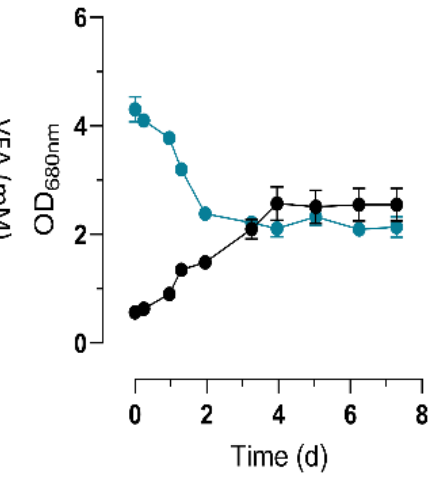

(b)

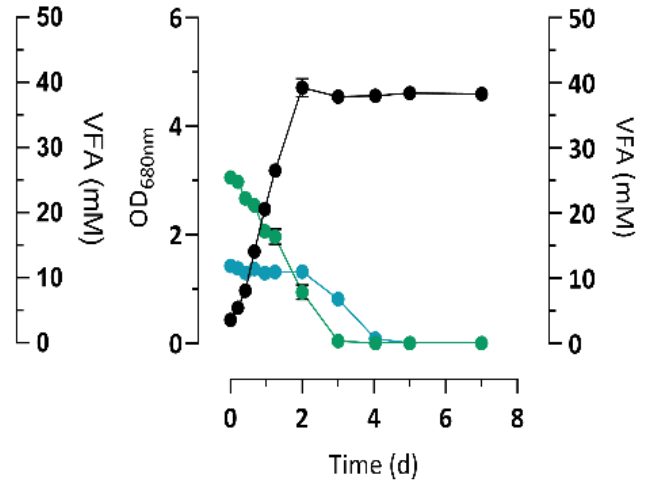

(c)

Figure 1. Monitoring of the growth $(\bullet)$ and VFA consumption in cultures of Rs. rubrum S1H cultivated with (a) propionate $(\bullet),(\mathbf{b})$ butyrate $(\bullet)$, or a mixture of both VFAS $(\mathbf{c})$, supplemented with $3 \mathrm{mM}$ of bicarbonate. Data presented are averages of 5 biological replicates with standard deviations.

Surprisingly, while the supplementation of $3 \mathrm{mM}$ of bicarbonate was not sufficient for complete assimilation of the $124 \mathrm{mM}$ of net equivalent carbon coming from propionate or butyrate (Figure 1a,b), when they were used as sole carbon sources, they were totally consumed by the nominal $3 \mathrm{mM}$ bicarbonate present in the culture medium when provided as a mixture of VFAS $_{S}$ (Figure 1c). This observation tended to indicate changes in the assimilation mechanism of propionate and butyrate when they are supplied together or individually. The presence of a cosubstrate also seems to induce faster growth of the biomass and faster removal of the carbon source (Figure 2), indicating a possible synergic effect due to the presence of several VFAS.

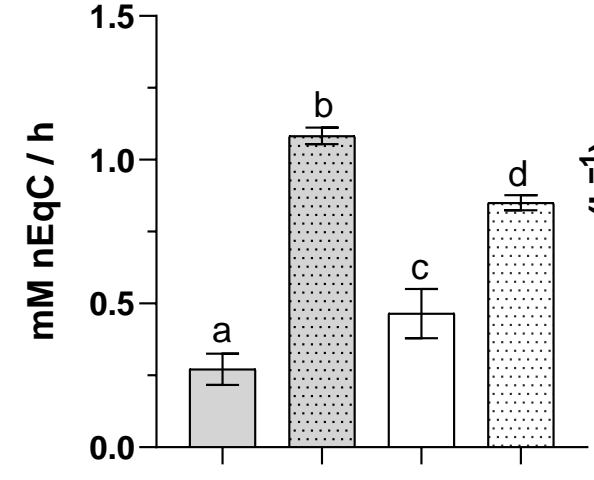

(a)

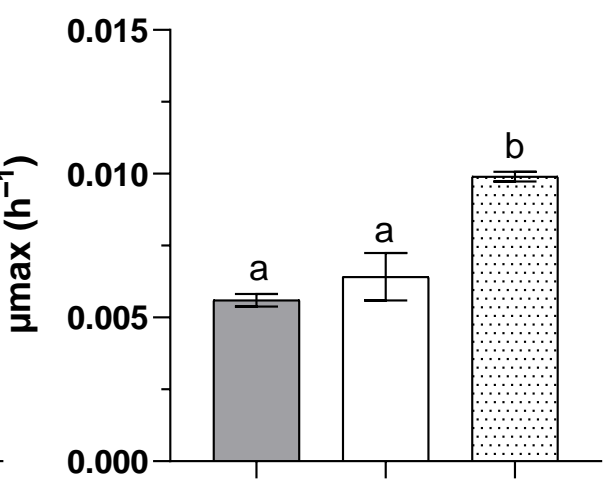

(b)

Figure 2. Carbon source consumption rate (a) and biomass growth rate (b) of Rs. rubrum grown using propionate $(\square)$, butyrate $(\square)$, or a mixture of these VFA $(\square)$ as the carbon source $(n=5)$. The letters a-d indicate statistically significant difference.

\subsection{Proteomic Analysis of Rs. rubrum Cultivated on a Propionate and Butyrate Mixture}

To better understand the differences in propionate and butyrate assimilation when supplied separately or as a mixture, we compared the proteomes of the biomass obtained with butyrate and propionate as sole carbon sources, as well as under binary mixture conditions, at different phases of the growth curve, i.e., during the early propionate assimilation phase and the late butyrate assimilation phase (Figure S1). In order to ensure continuous and complete assimilation of butyrate and propionate when provided as sole organic carbon sources, culture mediums were supplemented with $50 \mathrm{mM}$ of bicarbonate. To ensure proper comparison of the proteomic data obtained in the binary mixture and with butyrate and propionate only, the binary mixture was similarly performed with $50 \mathrm{mM}$ 
bicarbonate supplementation. A total of 1891 proteins were identified and quantified (false discovery rate below 1\%); these proteomic data covered about half of the theoretical proteome of Rs. rubrum S1 (ATCC1170, 3836 entries in Uniprot). Only proteins identified with more than 2 peptides and with significant $(p$-value $<0.05)$ and biologically relevant $(<0.66$ or $>1.5)$ fold changes were further considered. The complete data set is available in the Supporting Materials (Table S1).

\subsection{Propionate and Butyrate Photoassimilation by Rs. rubrum When Present as the Sole Carbon Source}

To clarify the impact of the presence of butyrate and propionate on their respective assimilation, it was necessary to first determine which proteins were specific to the assimilation of propionate or butyrate when supplied separately. We, thus, focused on proteins presenting a differential abundance between butyrate only and propionate only. Among the 277 proteins differentially regulated between these conditions, 135 presented fold changes lower than 0.66 (proteins more abundant under propionate condition) and 142 presented fold changes higher than 1.5 (proteins more abundant under butyrate conditions). The proteins discussed in the following paragraph are presented in Table 1.

Table 1. Differential expression of proteins in Rhodospirillum rubrum cultivate with propionate, butyrate, or a mixture of both.

* The fold change is the ratio of the abundance of a protein under butyrate conditions to the abundance under propionate conditions. ${ }^{* *}$ The fold change corresponds to the ratio of the abundance of a protein during the assimilation phase of butyrate and its abundance during the propionate assimilation phase under mixture conditions. ${ }^{* *}$ No. of identified peptides represents the number of peptides used for quantification.

\begin{tabular}{|c|c|c|c|c|c|c|c|}
\hline $\begin{array}{l}\text { Uniprot } \\
\text { Accession } \\
\text { Number }\end{array}$ & Locus Tag & $\begin{array}{l}\text { Fold Change } \\
\text { (Butyrate/ } \\
\text { Propioanate) }\end{array}$ & P-Value & $\begin{array}{l}\text { Fold Change (Mix } \\
\text { Butyrate/ } \\
\text { Mix Propionate) } * *\end{array}$ & P-Value & $\begin{array}{c}\text { No. of } \\
\text { Identified } \\
\text { Peptides }\end{array}$ & Description \\
\hline \multicolumn{8}{|c|}{ Methylmalonyl-CoA pathway } \\
\hline $\operatorname{tr} \mid \mathrm{Q} 2 \mathrm{RYD} 8$ & Rru_A0052 & 0.63 & $3.41 \times 10^{-2}$ & 1.81 & $9.50 \times 10^{-3}$ & 5 & Biotin carboxylase \\
\hline tr I Q2RYD7 & Rru_A0053 & 0.83 & $8.70 \times 10^{-2}$ & 1.81 & $4.95 \times 10^{-2}$ & 6 & Carboxyl transferase \\
\hline $\operatorname{tr} \mid \mathrm{Q} 2 \mathrm{RU} 23$ & Rru_A1572 & 1.30 & $3.49 \times 10^{-1}$ & $\begin{array}{l}1.01 \\
1.67\end{array}$ & $1.00 \times 10^{-2}$ & 5 & Methylmalonyl-CoA epimerase \\
\hline tr I Q2RRG6 & Rru_A2479 & 0.72 & $8.21 \times 10^{-2}$ & 1.75 & $9.56 \times 10^{-2}$ & 6 & Methylmalonyl-CoA mutase \\
\hline $\operatorname{tr} \mid$ Q2RRG5 & Rru_A2480 & 0.61 & $3.43 \times 10^{-2}$ & 1.56 & $1.37 \times 10^{-3}$ & 6 & Methylmalonyl-CoA mutase \\
\hline \multicolumn{8}{|c|}{ Methilcitrate cycle } \\
\hline $\operatorname{tr} \mid \mathrm{Q} 2 \mathrm{RRX7}$ & Rru_A2318 & 0.76 & $4.60 \times 10^{-1}$ & 0.47 & $1.22 \times 10^{-2}$ & 4 & 2-methylcitrate dehydratase \\
\hline $\operatorname{tr} \mid \mathrm{Q} 2 \mathrm{R} \times 6$ & Rru_A2319 & 0.25 & $1.61 \times 10^{-1}$ & 0.53 & $3.96 \times 10^{-2}$ & 5 & Citrate synthase \\
\hline $\operatorname{tr} \mid \mathrm{Q} 2 \mathrm{RRX} 5$ & Rru_A2320 & 1.07 & $8.75 \times 10^{-1}$ & 0.66 & $3.42 \times 10^{-1}$ & 2 & 2.3-dimethylmalate lyase \\
\hline \multicolumn{8}{|c|}{ Ethylmalonyl-CoA pathway } \\
\hline $\operatorname{tr} \mid \mathrm{Q} 2 \mathrm{RXX3}$ & Rru_A0217 & 1.97 & $1.38 \times 10^{-2}$ & 0.72 & $4.46 \times 10^{-1}$ & 6 & Citrate lyase \\
\hline $\operatorname{tr} \mid \mathrm{Q} 2 \mathrm{R} X \mathrm{R} 7$ & Rru_A0273 & 0.556 & $1.00 \times 10^{-1}$ & 0.74 & $7.70 \times 10^{-3}$ & 5 & 3-oxoacyl-eductase \\
\hline $\operatorname{tr} \mid \mathrm{Q} 2 \mathrm{RV} 43$ & Rru_A1201 & 1.48 & $8.73 \times 10^{-4}$ & 1.34 & $3.48 \times 10^{-1}$ & 6 & MaoC-like dehydratase \\
\hline $\operatorname{tr} \mid \mathrm{Q} 2 \mathrm{RTB} 0$ & Rru_A1835 & 1.97 & $1.43 \times 10^{-2}$ & 1.49 & $1.41 \times 10^{-1}$ & 4 & Butyryl-CoA dehydrogenase \\
\hline $\operatorname{tr} \mid \mathrm{Q} 2 \mathrm{RT} 18$ & Rru_A1927 & 0.35 & $1.60 \times 10^{-2}$ & 2.33 & $9.63 \times 10^{-3}$ & 6 & Acetyl-CoA hydrolase \\
\hline $\operatorname{tr}$ IQ2RPS1 & Rru_A3079 & 1.49 & $7.00 \times 10^{-3}$ & 1.05 & $3.24 \times 10^{-1}$ & 6 & 3-hydroxyacyl-CoA dehydrogenase \\
\hline $\operatorname{tr}$ IQ2RQ36 & Rru_A2964 & 1.03 & $8.16 \times 10^{-1}$ & 0.87 & $3.28 \times 10^{-1}$ & 2 & MaoC-like dehydratase \\
\hline $\operatorname{tr} \mid \mathrm{Q} 2 \mathrm{RPT} 8$ & Rru_A3062 & 2.62 & $2.59 \times 10^{-2}$ & 1.59 & $1.68 \times 10^{-1}$ & 5 & Methylmalonyl-CoA mutase \\
\hline $\operatorname{tr} \mid \mathrm{Q} 2 \mathrm{RPT} 7$ & Rru_A3063 & 6.17 & $4.78 \times 10^{-2}$ & 0.76 & $2.34 \times 10^{-1}$ & 6 & Crotonyl-CoA reductase \\
\hline $\operatorname{tr}$ IQ2RPT6 & Rru_A3064 & 2.23 & $4.96 \times 10^{-2}$ & 1.22 & $5.03 \times 10^{-1}$ & 6 & Isovaleryl-CoA dehydrogenase \\
\hline $\operatorname{tr} \mid \mathrm{Q} 2 \mathrm{RSZ} 9$ & Rru_A1946 & 2.63 & $5.35 \times 10^{-3}$ & 1.18 & $3.40 \times 10^{-1}$ & 5 & Acetyl-CoA C-acetyltransferase \\
\hline $\operatorname{tr} \mid \mathrm{Q} 2 \mathrm{RMQ0}$ & Rru_A3801 & 1.05 & $5.90 \times 10^{-1}$ & 0.93 & $6.26 \times 10^{-1}$ & 6 & Short chain enoyl-CoA hydratase \\
\hline \multicolumn{8}{|c|}{ Methylbutanoyl-CoA pathway } \\
\hline $\operatorname{tr} \mid \mathrm{Q} 2 \mathrm{R} X 73$ & Rru_A0467 & 1.25 & $3.69 \times 10^{-1}$ & 1.66 & $1.51 \times 10^{-2}$ & 6 & Acetolactate synthase. large subunit \\
\hline $\operatorname{tr} \mid \mathrm{Q} 2 \mathrm{R} X 72$ & Rru_A0468 & 0.99 & $8.96 \times 10^{-1}$ & 1.05 & $7.30 \times 10^{-1}$ & 3 & Acetolactate synthase. small subunit \\
\hline $\mathrm{sp} \mid \mathrm{Q} 2 \mathrm{RX71}$ & Rru_A0469 & 1.58 & $6.27 \times 10^{-2}$ & 2.02 & $4.40 \times 10^{-3}$ & 6 & Ketol-acid reductoisomerase \\
\hline $\operatorname{tr} \mid \mathrm{Q} 2 \mathrm{RX} 33$ & Rru_A0508 & 1.40 & $4.91 \times 10^{-1}$ & 1.07 & $9.34 \times 10^{-1}$ & 1 & Aminotransferase \\
\hline $\operatorname{tr}$ I Q2RWJ5 & Rru_A0696 & 0.47 & $9.37 \times 10^{-2}$ & 1.10 & $8.65 \times 10^{-1}$ & 1 & RNA methyltransferase \\
\hline sp |Q2RV55 & Rru_A1189 & 0.92 & $4.49 \times 10^{-1}$ & 1.12 & $8.59 \times 10^{-2}$ & 5 & $\begin{array}{l}\text { 3-isopropylmalate dehydratase } \\
\text { large subunit }\end{array}$ \\
\hline sp |Q2RV54 & Rru_A1190 & 1.06 & $5.53 \times 10^{-1}$ & 1.49 & $2.54 \times 10^{-1}$ & 6 & $\begin{array}{l}\text { 3-isopropylmalate dehydratase } \\
\text { small subunit }\end{array}$ \\
\hline sp |Q2RV53 & Rru_A1191 & 0.83 & $8.25 \times 10^{-2}$ & 0.87 & $1.96 \times 10^{-1}$ & 6 & 3-isopropylmalate dehydrogenase \\
\hline sp | Q2RTF9 & Rru_A1786 & 0.92 & $4.73 \times 10^{-1}$ & 1.04 & $6.44 \times 10^{-1}$ & 6 & Dihydroxy-acid dehydratase \\
\hline $\operatorname{tr} \mid \mathrm{Q} 2 \mathrm{RTB} 1$ & Rru_A1834 & 1.16 & $4.20 \times 10^{-1}$ & 1.26 & $9.54 \times 10^{-2}$ & 2 & Enoyl-CoA hydratase/isomerase \\
\hline $\operatorname{tr} \mid \mathrm{Q} 2 \mathrm{RT} 00$ & Rru_A1945 & 2.17 & $4.24 \times 10^{-3}$ & 1.11 & $5.24 \times 10^{-1}$ & 5 & Short-chain dehydrogenase/reductase \\
\hline $\operatorname{tr}$ IQ2RSZ7 & Rru_A1948 & 3.14 & $3.47 \times 10^{-2}$ & 0.91 & $7.65 \times 10^{-1}$ & 2 & Isovaleryl-CoA dehydrogenase \\
\hline $\operatorname{tr} \mid \mathrm{Q} 2 \mathrm{RSW} 8$ & Rru_A1977 & 4.86 & $3.21 \times 10^{-2}$ & 3.13 & $1.81 \times 10^{-2}$ & 2 & Pyruvate ferredoxin/flavodoxin \\
\hline $\operatorname{tr} \mid \mathrm{Q} 2 \mathrm{RSW7}$ & Rru_A1978 & 3.32 & $2.41 \times 10^{-2}$ & 2.02 & $4.90 \times 10^{-2}$ & 6 & $\begin{array}{l}\text { oxidoreductase } \\
\text { Indolepyruvate ferredoxin oxidoreductase }\end{array}$ \\
\hline $\operatorname{tr} \mid \mathrm{Q} 2 \mathrm{RS} 72$ & Rru_A2223 & 0.79 & $2.10 \times 10^{-1}$ & 1.87 & $4.90 \times 10^{-2}$ & 6 & $\begin{array}{c}\text { 2-keto-4-methylthiobutyrate } \\
\text { aminotransferase }\end{array}$ \\
\hline sp |Q53046 & Rru_A2398 & 1.79 & $4.72 \times 10^{-2}$ & 11.31 & $4.22 \times 10^{-2}$ & 6 & Pyruvate-flavodoxin oxidoreductase \\
\hline
\end{tabular}


Table 1. Cont.

\begin{tabular}{|c|c|c|c|c|c|c|c|}
\hline $\begin{array}{l}\text { Uniprot } \\
\text { Accession } \\
\text { Number }\end{array}$ & Locus Tag & $\begin{array}{l}\text { Fold Change } \\
\text { (Butyrate/ } \\
\text { Propioanate) * }\end{array}$ & P-Value & $\begin{array}{c}\text { Fold Change (Mix } \\
\text { Butyrate/ } \\
\text { Mix Propionate) }\end{array}$ & P-Value & $\begin{array}{c}\text { No. of } \\
\text { Identified } \\
\text { Peptides }\end{array}$ & Description \\
\hline \multicolumn{8}{|c|}{ Tricarboxylic carbon cycle } \\
\hline $\operatorname{tr} \mid \mathrm{Q} 2 \mathrm{RV} 44$ & Rru_A1200 & 1.87 & $2.59 \times 10^{-3}$ & 1.53 & $1.06 \times 10^{-1}$ & 5 & Citrate lyase \\
\hline $\operatorname{tr} \mid \mathrm{Q} 2 \mathrm{RV} 40$ & Rru_A1204 & 1.82 & $1.58 \times 10^{-2}$ & 1.55 & $4.84 \times 10^{-3}$ & 6 & MaoC-like dehydratase \\
\hline $\operatorname{tr} \mid \mathrm{Q} 2 \mathrm{RV} 39$ & Rru_A1205 & 1.98 & $3.64 \times 10^{-2}$ & 1.54 & $4.01 \times 10^{-2}$ & 5 & MaoC-like dehydratase \\
\hline $\operatorname{tr}$ I Q2RT66 & Rru_A1879 & 0.82 & $2.98 \times 10^{-1}$ & 1.03 & $7.28 \times 10^{-1}$ & 6 & Dihydrolipoamide acetyltransferase \\
\hline $\operatorname{tr}$ IQ2RT65 & Rru_A1880 & 0.85 & $3.22 \times 10^{-1}$ & 1.08 & $3.59 \times 10^{-1}$ & 6 & Pyruvate dehydrogenase beta subunit \\
\hline $\operatorname{tr}$ IQ2RT64 & Rru_A1881 & 1.00 & $9.92 \times 10^{-1}$ & 0.98 & $9.21 \times 10^{-1}$ & 4 & Pyruvate dehydrogenase \\
\hline $\operatorname{tr} \mid$ Q2RS89 & Rru_A2206 & 0.79 & $1.46 \times 10^{-1}$ & 1.72 & $1.14 \times 10^{-3}$ & 6 & Fumarase \\
\hline $\operatorname{tr} \mid \mathrm{Q} 2 \mathrm{RNT} 2$ & Rru_A3419 & 1.88 & $6.88 \times 10^{-3}$ & 3.02 & $1.35 \times 10^{-3}$ & 6 & Phosphoenolpyruvate carboxykinase \\
\hline \multicolumn{8}{|c|}{ Calvin-Benson-Bassham cycle } \\
\hline sp |Q2RRP5 & Rru_A2400 & 4.20 & $3.45 \times 10^{-2}$ & 2.68 & $1.86 \times 10^{-1}$ & 6 & Ribulose bisphosphate carboxylase \\
\hline \multicolumn{8}{|c|}{ Reverse Tricarboxylic carbon cycle } \\
\hline $\operatorname{tr} \mid$ Q2RQS7 & Rru_A2721 & 0.96 & $9.26 \times 10^{-1}$ & 1.52 & $1.48 \times 10^{-1}$ & 2 & 2-oxoglutarate synthase. alpha subunit \\
\hline $\operatorname{tr} \mid \mathrm{Q} 2 \mathrm{RQS6}$ & Rru_A2722 & 0.99 & $9.75 \times 10^{-1}$ & 1.50 & $2.75 \times 10^{-2}$ & 5 & 2-oxoglutarate synthase, beta subunit \\
\hline
\end{tabular}

The data obtained were in favor of the assimilation of propionate through its conversion in succinyl-CoA via a methylmalony-CoA intermediate (Figure 3), confirming the previous reports [18]. This pathway requires five enzymes (Rru_A0052, Rru_A0053, Rru_A1572, Rru_A2480, Rru_A1927), among which three were significantly more abundant in propionate conditions than in butyrate conditions: the biotin carboxylase (Rru_A0052, fold change: 0.63), the methylmalonyl-CoA mutase (Rru_A2480, fold change: 0.61), and the succinyl-CoA transferase (Rru_A1927, fold change: 0.35). The carboxyl transferase, Rru_A0053, was also quantified in slightly higher abundance in propionate conditions (fold change: $0.83, p$-value $<0.05$ ), but did not reach the biological relevance threshold used in this study. Regarding proteins presenting a higher abundance in butyrate conditions, the data were consistent with a previous proteomic analysis carried out in our lab, showing assimilation of butyrate through both the EMC and MBC pathways (Figure 3) [19]. Indeed, the butyryl-CoA dehydrogenase (Rru_A1835, fold change: 1.97) involved in the conversion of butyryl-CoA to crotonyl-CoA was upregulated under butyrate conditions, as was the cluster of key enzymes involved in the EMC pathway (Rru_A3062, fold change: 2.62; Rru_A3063, fold change: 6.17; Rru_3064, fold change: 2.23) (Table 1).

A higher abundance of proteins involved in the newly proposed MBC pathway, which combined branched-chain amino acid biosynthesis and degradation, was also noticed. The biosynthesis of BCAA responsible for the conversion of acetyl-CoA into (S)-3-methyl2-oxopentanoate was not significantly upregulated under butyrate conditions; however, the indole oxidoreductase (Rru_A1977 and Rru_A1978) that connect the BCAA biosynthesis pathway to the BCAA degradation pathway was more abundant under butyrate conditions than propionate conditions (Rru_A1977, fold change: 4.86; Rru_A1978, fold change: 3.32 ), with the same observation being made for the cluster of enzymes involved in the isoleucine degradation pathway (Rru_A1945, fold change: 2.17; Rru_A1946, fold change: 2.63; Rru_A1948, fold change: 3.14) (Table 1). These results support the previous hypothesis that propionate is assimilated through the methylmalonyl-CoA pathway, while butyrate is assimilated through the EMC and MBC pathways when these carbon sources are present separately.

\subsection{Photoassimilation of Butyrate and Propionate by Rs. rubrum When Present as a Mixture}

\subsubsection{Methylcitrate Cycle}

In contrast to previous observations, the number of enzymes involved in the conversion of propionyl-CoA in succinyl-CoA via the methylmalonyl-CoA intermediate pathway was significantly higher during the butyrate assimilation phase (Table 1). Comparing the abundances of these enzymes between propionate-only conditions and the propionate assimilation phase under binary mixture conditions also revealed a higher abundance when propionate was the only carbon source (Table S1). These observations suggested a lower dependency of propionate assimilation on the methylmalonyl-CoA pathway when butyrate was simultaneously present in the medium. On the other hand, the citrate syn- 
thase (Rru_A2319) and the 2-methylcitrate dehydratase (Rru_A2318) were more abundant during the propionate assimilation phase under binary mixture conditions (Rru_A2318, fold change: 0.47; Rru_A2319, fold change: 0.53) (Figure 3). The 2,3-dimethylmalate lyase (Rru_2320) also presented a higher abundance during the propionate assimilation phase (fold change: 0.66 ), although this fold change was not statistically significant ( $p$-value: 0.34). The citrate synthase and 2-methylcitrate dehydratase were also more abundant when propionate was assimilated in a binary mixture than when this VFA was provided as a sole carbon source (Rru_A2318, fold change: 0.61; Rru_A2319, fold change: 0.52). These enzymes are involved in the methylcitrate cycle, a metabolic pathway involved the assimilation of propionate [27]. The data, thus, indicate a potential change in the metabolic pathway used for propionate assimilation when butyrate is present in the medium, with a higher dependency on the methylcitrate cycle.

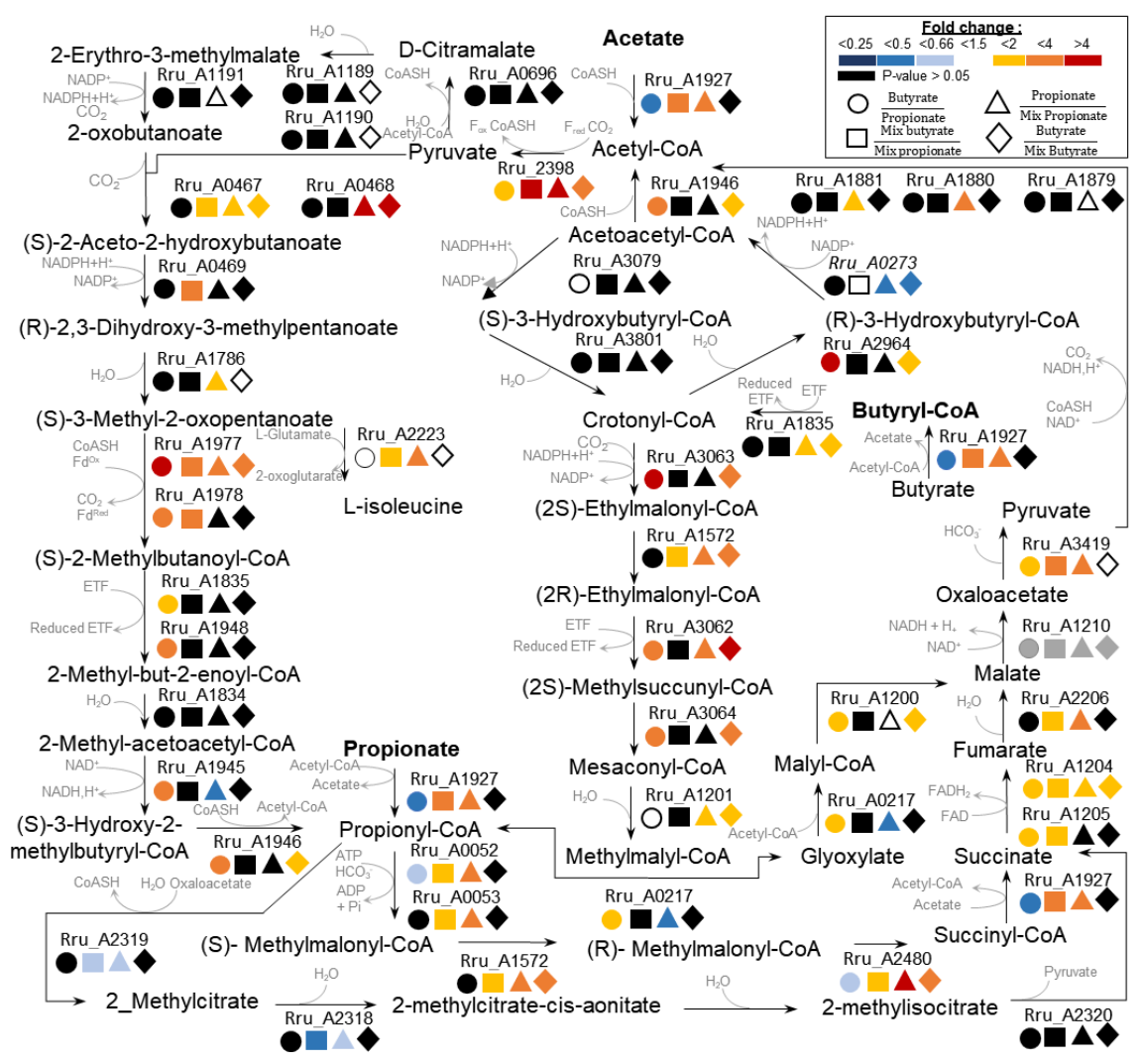

Figure 3. Schematic representation of the metabolic pathways highlighted through differential proteomic analysis $(n=5)$. The different colors indicate the fold changes: white, biologically nonrelevant fold change (fold change value between 0.66 and 1.5); black, fold change not significant ( $p$-value > 0.05); grey, protein not identified.

\subsubsection{EMC and MBC Pathways}

Under butyrate only conditions, proteomic data indicated that both EMC- and MBCpathway-related enzymes were more abundant, as mentioned above. Interestingly, under binary mixtures condition, only enzymes of the MBC pathway were still more abundant during the butyrate assimilation phase. Indeed, the cluster of key enzymes of the EMC pathway (Rru_A3062, Rru_A3063, Rru_A3064) was no longer upregulated during the assimilation of butyrate (Table 1). Comparing butyrate only conditions with the butyrate assimilation phase under binary mixture conditions, these three enzymes were observed to be significantly more abundant under the butyrate only conditions (Rru_A3062, fold change: 4.48; Rru_A3063, fold change: 3.12; Rru_A3064, fold change: 2.41) (Table S1). These data suggested a lower dependency of butyrate assimilation on the EMC pathway when Rs. rubrum was grown on a binary mixture. On the other hand, some of the enzymes 
involved in the first part of the MBC pathway and corresponding to the BCAA biosynthesis pathway (acetolactate synthase large subunit (Rru_A0467), pyruvate-flavodoxin oxidoreductase, NiFJ (Rru_A2398), and ketol acid reductoisomerase (Rru_A0469)) were observed in higher abundance during the butyrate assimilation phase under binary mixture conditions. On the other hand, these enzymes were systematically more abundant when butyrate and propionate were present separately than during their respective assimilation phase in the binary mixture. We recently suggested that in Rs. Rubrum, transient isoleucine accumulation could be an electron sinking mechanism, with its synthesis involved in consumption of the reducing equivalent $[19,28]$.

Altogether, these observations suggest that the BCAA synthesis pathway is more active when propionate and butyrate are present separately rather than in a mixture and potentially that more isoleucine is produced under these conditions as well. To test this hypothesis, we monitored the activity of the acetolactate synthase in cells obtained in butyrate and propionate only conditions and from the binary mixture after 24 and $72 \mathrm{~h}$ of cultivation. The acetolactate activity per $\mathrm{mg}$ of total proteins was significantly higher in samples obtained at 72 than $24 \mathrm{~h}$ (Figure 4) in all three conditions and significantly higher at $72 \mathrm{~h}$ in propionate and butyrate only conditions than in the binary mixture. Since proteomic samples from propionate and butyrate only conditions were collected at 56 and $48 \mathrm{~h}$, respectively (Figure S1), the results of this activity test agree with the proteomic data. The potential activity of the BCAA synthesis pathway is effectively increased at later stages of the exponential growth phase, but also in propionate and butyrate only conditions as compared to the binary mixture after $72 \mathrm{~h}$ of culture.

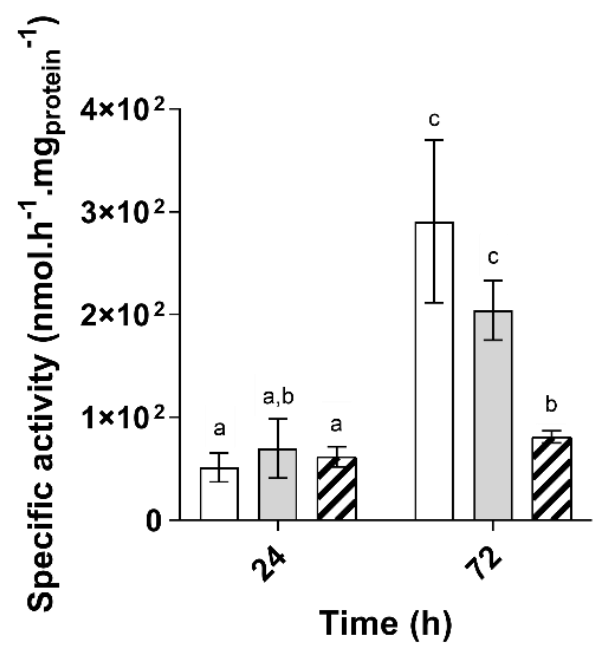

Figure 4. Differential expression of the enzymatic activity of Rs. rubrum acetolactate synthase in culture using propionate $(\square)$, butyrate $(\square)$, or a mixture of VFAS in culture supplemented with $50 \mathrm{mM}$ of bicarbonate $(\boldsymbol{Q})(n=5)$. The letters a-c indicate statistically significant difference.

3.3.3. Redox Homeostasis in Rs. rubrum When Butyrate and Propionate Are Used as Sole Organic Carbon Sources or as a Mixture

During photoheterotrophic growth, the oxidative pathways converting reduced organic substrates into biosynthetic precursors produced ample reducing power. To maintain the central metabolic flow, a large amount of the produced reducing equivalent must be recycled through an electron sinking pathway. Several redox balancing reactions have been proposed to fulfil this function, potentially including the synthesis of isoleucine described previously [28].

Carbon dioxide fixation is probably the most common reaction used by PNSB to maintain the pool of oxidized electron carriers. The Calvin-Benson-Bassham cycle (CBB) has been extensively described as the key pathway for carbon dioxide fixation through the activity of Rubisco (Rru_A2400) [29]. Rubisco was highly upregulated under butyrate only conditions in comparison with propionate only conditions (fold change: 4.20); however, the 
upregulation was less important (fold change: 2.68 ) and not significant ( $p$-value: 0.18 ) between the butyrate assimilation phase and the propionate assimilation phase under binary mixture conditions. In addition, while there was no significant change in the abundance of Rubisco under butyrate only conditions in comparison with the butyrate assimilation phase under binary mixture conditions ( $p$-value: 0.27 ), the enzyme was more abundant during the assimilation phase of propionate under binary mixture conditions in comparison with the propionate only conditions (Fold change: 0.36 ) (Table S1). It seems that the presence of butyrate, alone or in the binary mixture, systematically triggered an increased abundance of Rubisco, even during the propionate assimilation phase. Nonetheless, the activity of Rubisco and the CBB cycle is linked with the consumption of bicarbonate ions from the culture medium or $\mathrm{CO}_{2}$ produced by the catabolic reaction, with the former only participating in electron sinking. The lesser requirement for bicarbonate supplementation observed in binary mixtures suggested that alternative electron sinking mechanisms are activated in the presence of multiple VFA $\mathrm{V}_{\mathrm{S}}$ and that Rubisco upregulation in this context could be linked with a higher requirement for catabolic $\mathrm{CO}_{2}$ capture.

Other electron sinking pathways have been described in Rs. Rubrum, such as the reductive tricarboxylic acid cycle (rTCA) [30,31]. One of the key enzymes in this pathway, which is indicative of a functional reductive TCA, is the 2-oxoglutarate oxidoreductase (Rru_A2721, Rru_A2722). The 2-oxoglutarate oxidoreductase $\beta$ subunit (Rru_A2722) was significantly more abundant under mixture conditions during both propionate (fold change: 0.46 ) and butyrate (fold change: 0.22 ) assimilation phases than under butyrate or propionate only conditions; however, the abundance of the 2-oxoglutarate oxidoreductase $\alpha$ subunit (Rru_A2721) was not significantly different in any condition (Table S1). A functional rTCA cycle could be an additional electron sinking pathway allowing the cell to maintain its redox homeostasis; however, further experiments will be needed to confirm the activity of the rTCA under mixture conditions.

PHA production is very often proposed to also act as an electron sink [32]. We recently showed that this biopolymer accumulated in cells upon a sudden increase in light intensity, potentially in response to redox imbalance [33]. Proteomic data showed that PHA synthase (Rru_A2413) was highly upregulated under butyrate only conditions as compared to propionate only or binary mixture conditions and was invariant during the different assimilation phases in the binary mixture. To evaluate whether PHA also followed the same trend, we analyzed the PHA contents in cells obtained in all three conditions at 24 and $72 \mathrm{~h}$. As expected from the proteomic data, Rs. rubrum accumulated the largest amount of PHA under butyrate only conditions, both at 24 and $72 \mathrm{~h}$ (Figure 5a). In the binary mixture, on the other hand, the PHA content remained relatively low during the entire culture phase. This observation was similar to the data obtained for the additional mixture of VFA containing acetate, propionate, butyrate, and valerate, whereby the mixture always showed lower levels of PHA than under single organic carbon source conditions [20]. To determine whether low PHA accumulation in a binary mixture can be linked with the supplementation of the culture medium with $50 \mathrm{mM}$ of bicarbonate, we measured the PHA accumulation rates in cells grown in a binary mixture of propionate and butyrate supplemented with only $3 \mathrm{mM}$ of bicarbonate. When a slight increase was observed, the level of accumulation of PHA was still much lower than under butyrate only or acetate only conditions [33]. Consequently, we concluded that PHA was not involved in the electron sinking mechanism that allowed Rs. rubrum to assimilate propionate and butyrate when provided as a binary mixture without bicarbonate assimilation.

As we proposed that isoleucine accumulation could be linked with redox balancing and to test whether Rs. rubrum effectively accumulated isoleucine in the studied conditions, we monitored the intracellular isoleucine contents in all three conditions at 24 and $72 \mathrm{~h}$. The isoleucine content, as determined using LC-MS/MS and normalized based on the arginine content, was observed to be higher under butyrate conditions at $24 \mathrm{~h}$, confirming our previous observation [19] (Figure 5b). At $72 \mathrm{~h}$, the isoleucine content was also increased under propionate conditions, as expected from the proteomic analysis and acetolactate 
activity tests. Unexpectedly, an increase in the isoleucine content was observed in the binary mixture after $72 \mathrm{~h}$ of growth. No upregulation of the enzymes of the threoninedependent isoleucine synthesis pathway was observed under binary mixture conditions, although isoleucine could be produced by this alternative pathway.

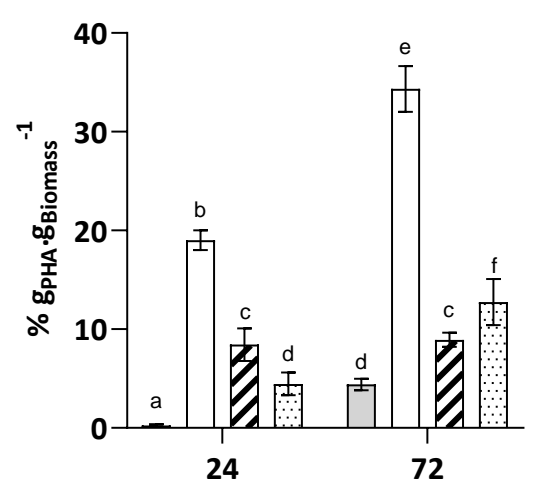

Time (h)

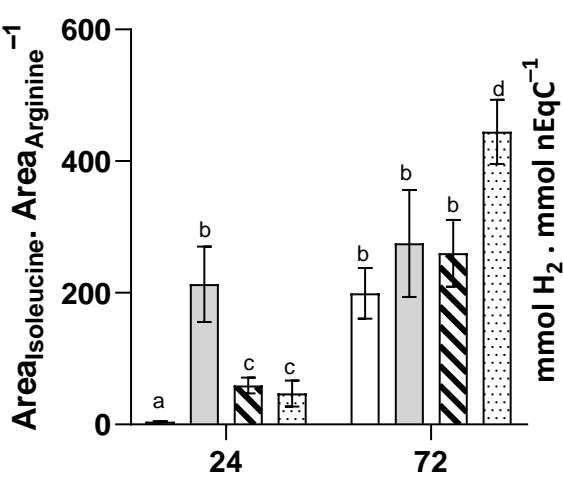

Time (h)

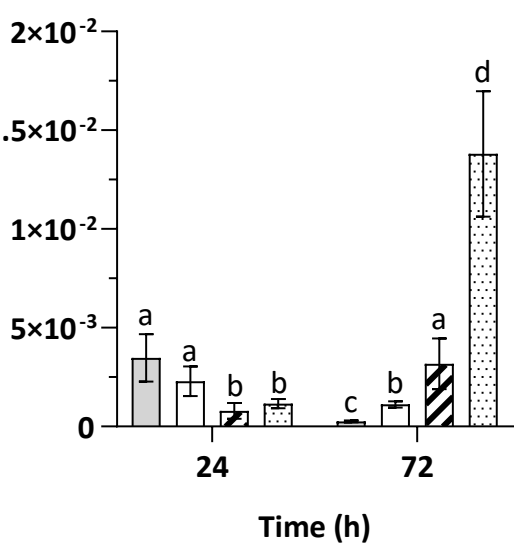

(c)

(a)

(b)

Figure 5. Differential accumulation of PHA (a), isoleucine (b), and dihydrogen (c) in Rs. rubrum culture using propionate $(\square)$, butyrate $(\square)$, or a mixture of these VFA as carbon sources supplemented with $50 \mathrm{mM}$ of bicarbonate $(\boldsymbol{\square})$ or $3 \mathrm{mM}$ of bicarbonate $(\mathrm{Q})(n=5)$. The letters a-f indicate statistically significant difference.

The accumulation of isoleucine could potentially be explained by the decreased abundance during the butyrate assimilation phase under mixture conditions in comparison with butyrate only conditions of the 2-oxoacid dehydrogenase family protein Rru_A1977, which is supposedly involved in linking isoleucine biosynthesis and degradation in the MBC assimilation pathway. It could be hypothesized that a lowered abundance of this pivotal enzyme of the isoleucine degradation, even without upregulation of the isoleucine synthesis pathway under binary mixture conditions, could explain the isoleucine accumulation under mixture conditions. When only $3 \mathrm{mM}$ of bicarbonate was provided, an increase in the isoleucine/arginine ratio was observed after $72 \mathrm{~h}$ of culture, suggesting that the isoleucine synthesis pathway is involved in redox homeostasis when propionate and butyrate are simultaneously use as carbon sources.

In some strains constitutively expressing nitrogenase, the production of $\mathrm{H}_{2}$ was shown to replace the CBB cycle as the electron sink [34]. The production of $\mathrm{H}_{2}$ was, therefore, quantified to see whether this redox balance mechanism occurred under mixture conditions. No major significant differences were observed when the culture was supplemented with $50 \mathrm{mM}$ of bicarbonate (Figure 5c); however, in a culture grown with a mixture of propionate and butyrate as the carbon source and supplemented with only $3 \mathrm{mM}$ of bicarbonate, a significant increase in $\mathrm{H}_{2}$ production was observed. The amount obtained was lower than the results found in the literature when malate was used as a carbon source [34]; however, this increase in $\mathrm{H}_{2}$ production when the availability of bicarbonate decreased could explain the lower requirement for bicarbonate supplementation under mixture conditions. Using our proteomic data set, it was not possible to determine the metabolic pathway involved in this process. Indeed, no changes in the abundance of proteins potentially involved in the production of $\mathrm{H}_{2}$ (hydrogenases, nitrogenase, or $\mathrm{CO}$-dehydrogenase) were observed in our proteomic analysis (Table S2). It has to be noted that as the carbon nitrogen ratio at the beginning of the culture was 3.54, the production of $\mathrm{H}_{2}$ likely occurred under nitrogen non-limiting conditions, which is highly unusual. Further analysis would be necessary to understand the synthesis of $\mathrm{H}_{2}$ in propionate-butyrate mixtures and to determine whether or not this phenomenon could be observed in other mixtures. 


\section{Discussion}

\subsection{Mixing VFAS Leads to Synergy in Their Assimilation}

The presence of several carbon sources appears to induce a synergic effect, as the highest $\mu_{\max }$ was observed under mixed VFAS conditions. A similar synergic effect was reported for photosynthetic cultures of a consortium of purple bacteria, whereby combinations of acetate with butyrate or propionate increased the uptake rates of VFA [4]. This phenomenon could result from the fact that with such mixtures, the biosynthesis of some precursors might be facilitated. For example, the balance of acetyl-CoA synthesis from butyryl-CoA is 0.5 Butyryl-CoA + 0.5 $\mathrm{ETF}_{\text {oxy }}+0.5 \mathrm{NADP}^{+} \rightarrow$ acetyl-CoA $+0.5 \mathrm{ETF}_{\text {red }}+0.5 \mathrm{NADPH}, \mathrm{H}^{+}$; whereas from propionyl-CoA, the balance is propionyl-CoA $+\mathrm{GDP}+\mathrm{Pi}+\mathrm{FAD}^{+}+2 \mathrm{NAD}^{+}+$ $\mathrm{H}_{2} \mathrm{O} \rightarrow \mathrm{GTP}+2 \mathrm{NADPH}, \mathrm{H}^{+}+\mathrm{FADH}_{2}+\mathrm{CO}_{2}+$ acetyl-CoA. Our proteomic analysis highlighted the use of the methylcitrate cycle for the assimilation of propionate in the presence of butyrate, whereas under propionate only conditions the methylmalonyl-CoA pathway seemed to be used. Referring to the pyruvate production, while the balance of the methylcitrate cycle is 1 propionyl-CoA $+2 \mathrm{H}_{2} \mathrm{O}+\mathrm{FAD}^{+}+\mathrm{NAD}^{+}+\mathrm{ATP} \rightarrow 1$ Pyruvate $+\mathrm{FADH}_{2}+\mathrm{NADH}+\mathrm{H}^{+}$, the balance of the production of pyruvate via the methylmalonyl-CoA is 1 propionyl$\mathrm{CoA}+\mathrm{FAD}^{+}+\mathrm{NAD}^{+} \mathrm{H}_{2} \mathrm{O} \rightarrow \mathrm{CoASH}+\mathrm{FADH}_{2}+\mathrm{NADH}+\mathrm{H}^{+}+1$ pyruvate. Based on the balance of each pathway, they seem to be equivalent regarding the $\mathrm{CO}_{2}$ or reducing power consumption; however, the difference between these assimilation routes could be due to the lower energetic cost of the methylcitrate cycle and the recovery of the energy needed to activate propionate by the transfer of the CoASH. In the methylmalonyl-CoA pathway, during the conversion of succinyl-CoA in succinate, the CoASH is supposedly transferred to acetate to form acetyl-CoA, while during the conversion of propionyl-CoA in methylcitrate, the CoASH seems to be hydrolyzed and is not recovered. This difference might make the methylcitrate pathway less efficient at this level. On the other hand, producing acetyl-CoA can be achieved more easily from butyryl-CoA than through methylmalonyl-CoA when propionate and butyrate are mixed, explaining the faster growth under mixed conditions. Another possible explain for the use of the methylcitrate cycle under mixture conditions could be the lower concentration of propionate. A previous study on Burkholderia sp. showed a change in the assimilation pathway used for propionate assimilation between a methylcitrate intermediate and the acrylate pathway according to the level of propionate in the medium [35]. At a low level, propionate was assimilated via the methylcitrate pathway, while at a high level its assimilation involved the acrylate pathway [35,36]. A similar phenomenon could occur under mixture conditions when the level of propionate is lower than under propionate-only conditions.

\subsection{Mixing VFAs Abolishes the Need for Bicarbonate Supplementation for the Assimilation of Reduced Substrates}

In the absence of oxygen or other electron acceptors, oxidative phosphorylation cannot recycle all of the reduced cofactors produced during oxidative reactions; hence, the recycling of cofactors must occur via alternative pathways, described as electron sinks. Several redox balancing reactions have been proposed, although carbon dioxide fixation is probably the most common reaction used to maintain the pool of oxidized electron carriers and is strongly related to the redox state of the carbon source; the more reduced the carbon source, the higher the requirement for $\mathrm{CO}_{2}$ fixation, potentially requiring exogenous $\mathrm{CO}_{2}$ supplementation [37]. Surprisingly, while the supplementation of the culture medium with bicarbonate is a requirement observed with the most reduced carbon sources, such as propionate [38], butyrate [38], and to a lesser extent acetate [16,39], we recently showed that this supplementation is no longer required if Rs. rubrum is photoheterotrophically grown with mixtures of those three VFAs [20]. It could be hypothesized that the initial amount of bicarbonate available in the culture medium $(3 \mathrm{mM})$ might allow the complete assimilation of the lower amounts of propionate and butyrate provided as a mixture in this study; however, experiments carried out on butyrate and propionate used as sole carbon sources showed that the nominal $3 \mathrm{mM}$ of bicarbonate present in the mixture allowed 
the assimilation of less than $10 \mathrm{mM}$ of propionate or $15 \mathrm{mM}$ of butyrate, while the initial $20.6 \mathrm{mM}$ and $15.5 \mathrm{mM}$ of propionate and butyrate, respectively, were entirely assimilated when used as a mixed carbon source [20].

The lower requirement of bicarbonate supplementation could also be due to a change in the metabolic pathway involved in the assimilation of propionate and butyrate when they were simultaneously present $[35,36]$. For instance, the assimilation of butyrate in a binary mixture with propionate seemed to preferentially occur through the MBC pathway rather than the EMC pathway, with the latter not being particularly upregulated under these growth conditions. The EMC pathway consumes $\mathrm{CO}_{2}$, meaning carbon assimilation metabolism based on pathways that are less dependent on carbon dioxide, such as the MBC pathway, might be consistent with the observed lower need for bicarbonate supplementation under mixture conditions. Moreover, a kinetic proteomic analysis performed in butyrate showed that the abundances of most of the enzymes involved in the EMC pathway were relatively constant during the whole growth phase [19]. The absence of activation of this pathway is not likely to be related to an apparent "end of grow phase" phenotype but rather to the presence of propionate in the medium. Combining VFAs, therefore, seems to definitely impact the way they are assimilated.

\subsection{Mixing VFAs Changes the Mechanism Involved in the Redox Balance of the Cells}

As dependency on bicarbonate fixation is decreased under mixtures of propionate and butyrate, and as $\mathrm{CO}_{2}$ fixation is known to be one of the main redox balancing mechanisms in purple bacteria, one may ask how redox balancing occurs under such conditions. Alternative electron sinking mechanisms may be used, such as reverse TCA or PHA production.

One of the characteristic enzymes of the rTCA cycle, the 2-oxoglutarate oxidoreductase, was identified in the proteomic data set and quantified as having a higher abundance under mixture conditions compared to conditions involving either VFA as a single carbon source; however, although these may be the first results, the presence of this enzyme is not sufficient by itself and further analysis will be needed to support the hypothesis of an operational rTCA cycle during assimilation of a mixture of butyrate and propionate.

In Rs. rubrum growing on VFA, the production of PHA has been described as a possible redox balance mechanism. The production of hydroxybutyrate or hydroxyvalerate from propionate results in consumption of the redox equivalent; however, when production occurs from butyrate, PHA synthesis cannot play the role of an electron sink. It was hypothesized that under butyrate conditions, the PHA production can be used as a buffer zone to regulate the production of the reduced equivalent, making PHA synthesis a possible redox balance mechanism $[19,40]$. We recently linked the presence of propionate to a low level of PHA accumulation [20]. The presence of propionate in our mixture could, thus, potentially explain the lower PHA production observed under such conditions. The low PHA production rate in our binary mixture indicated that PHA production was not used here as a major electron sinking reaction and could not counterbalance the observed lower bicarbonate requirement.

Studies carried out on Rs. rubrum grown on VFAs have highlighted a possible involvement of branched-chain amino acid accumulation, in particular isoleucine, as a possible electron sink during Rs. rubrum growth on acetate and butyrate $[19,28,41]$. In a mutant culture lacking Rubisco, the addition of $\alpha$-ketoglutarate-derived amino acids inhibited the activity of the BCAA synthesis pathway and prevented the bacterial grow unless DMSO was added, indicating the involvement of this pathway in the redox balance maintenance of the cells [41]. Moreover, it was shown that in a culture of Rs. rubrum subjected to a rapid increase in light intensity, a phenomenon known to create redox stress [33], an increase in the isoleucine/arginine ratio occurred [28]. This indicated the importance of the BCAA synthesis pathway as a redox balance mechanism during the growth of Rs. rubrum under photoheterotrophic conditions. In culture supplemented with a mixture of propionate and butyrate and an initially low amount of carbonate $(3 \mathrm{mM})$, the measured level of isoleucine was significatively higher than that observed under butyrate conditions, indicating that 
isoleucine production could be involved in the bicarbonate-independent redox balancing observed under mixed condition.

Finally, the focus was shifted to $\mathrm{H}_{2}$ production, as it was demonstrated that $\mathrm{H}_{2}$ synthesis could replace the CBB cycle as an electron sink in strains of Rs. rubrum constitutively expressing nitrogenase $[29,34]$. Under mixture conditions, a significant increase in $\mathrm{H}_{2}$ production was observed when cultures were grown with a mixture of propionate and butyrate as the carbon source, especially when the medium was supplemented with only $3 \mathrm{mM}$ of bicarbonate. The ratio observed between the quantity of $\mathrm{H}_{2}$ produced per net equivalent of carbon assimilated was lower than the value found in the literature for this strain, especially when propionate or butyrate was used as the sole carbon source [34]; however, the presence of a nitrogen source in the medium could explain why $\mathrm{H}_{2}$ was only produced in small quantities. Further analysis would be required to better understand this increase in $\mathrm{H}_{2}$ production, especially in terms of the mechanism triggering this synthesis under nitrogen non-limiting conditions.

\section{Conclusions}

The use of mixed VFAs appears to have a synergistic effect on growth phenotypes. Moreover, it seems that the presence of a mixture of VFAs removes the requirement for carbonate supplementation to allow the assimilation of butyrate and propionate by triggering $\mathrm{H}_{2}$ production and isoleucine accumulation. The concomitant use of these diverse electron sinking pathways could be a first step toward understanding the reduced need for external electron acceptors under mixture conditions; however, the reason why these mechanisms are inefficient when only one VFA is used as a carbon source still requires further investigation. The results obtained in this study open the door to a better understanding and more efficient utilization of PNSB in biotech processes using VFAs.

Supplementary Materials: The following are available online at https:/ / www.mdpi.com/article/10 $.3390 /$ microorganisms9091996/s1: Figure S1: Monitoring of the growth and butyrate and propionate consumption rates in a culture of Rs. rubrum $\mathrm{S} 1 \mathrm{H}$ cultivated in propionate, butyrate, or a mixture of both, along with the proteomic sample times. Table S1: Full data set for the proteomic analysis carried out on Rs. Rubrum. Table S2: Differential expression of enzyme possibly involved in the $\mathrm{H} 2$ production.

Author Contributions: Conceptualization, B.L. and P.C.S.; methodology, A.T., R.O., Q.D.M., P.C.S. and B.L.; validation, P.C.S. and B.L.; formal analysis, P.C.S.; investigation, P.C.S. and Q.D.M.; resources, A.T., R.O., R.W. and B.L.; data curation, P.C.S.; writing-original draft preparation, P.C.S. and B.L.; writing-review and editing, L.D. and R.W.; visualization, B.L.; supervision, R.W., B.L. and L.D.; project administration, B.L. and L.D.; funding acquisition, B.L. and R.W. All authors have read and agreed to the published version of the manuscript.

Funding: This research was funded by the Belgian Fund for Scientific Research (Grand EquipmentF.R.S-FNRS) and the Concerted Research Action ARC project.

Institutional Review Board Statement: Not applicable.

Informed Consent Statement: Not applicable.

Data Availability Statement: Not applicable.

Acknowledgments: The Bioprofiling platform used for proteomic analysis was supported by the European Regional Development Fund and the Walloon Region, Belgium.

Conflicts of Interest: The authors declare no conflict of interests.

\section{References}

1. Garrity, G. Bergey's Manual ${ }^{\circledR}$ of Systematic Bacteriology: Volume Two: The Proteobacteria, Part A Introductory Essays; Springer Science \& Business Media: Berlin/Heidelberg, Germany, 2006; Volume 2, ISBN 0387280219.

2. Bengtsson, S.; Werker, A.; Christensson, M.; Welander, T. Production of polyhydroxyalkanoates by activated sludge treating a paper mill wastewater. Bioresour. Technol. 2008, 99, 509-516. [CrossRef] 
3. Kim, D.-H.H.; Lee, J.-H.H.; Hwang, Y.; Kang, S.; Kim, M.-S.S. Continuous cultivation of photosynthetic bacteria for fatty acids production. Bioresour. Technol. 2013, 148, 277-282. [CrossRef]

4. Fradinho, J.C.; Oehmen, A.; Reis, M.A.M.M. Photosynthetic mixed culture polyhydroxyalkanoate (PHA) production from individual and mixed volatile fatty acids (VFAs): Substrate preferences and co-substrate uptake. J. Biotechnol. 2014, $185,19-27$. [CrossRef]

5. Alloul, A.; Wuyts, S.; Lebeer, S.; Vlaeminck, S.E. Volatile fatty acids impacting phototrophic growth kinetics of purple bacteria: Paving the way for protein production on fermented wastewater. Water Res. 2019, 152, 138-147. [CrossRef] [PubMed]

6. Capson-Tojo, G.; Batstone, D.J.; Grassino, M.; Vlaeminck, S.E.; Puyol, D.; Verstraete, W.; Kleerebezem, R.; Oehmen, A.; Ghimire, A.; Pikaar, I. Purple phototrophic bacteria for resource recovery: Challenges and opportunities. Biotechnol. Adv. 2020, $43,107567$. [CrossRef] [PubMed]

7. Alloul, A.; Cerruti, M.; Adamczyk, D.; Weissbrodt, D.; Vlaeminck, S. Control tools to selectively produce purple bacteria for microbial protein in raceway reactors. BioRxiv 2020. [CrossRef]

8. Anterrieu, S.; Quadri, L.; Geurkink, B.; Dinkla, I.; Bengtsson, S.; Arcos-Hernandez, M.; Alexandersson, T.; Morgan-Sagastume, F.; Karlsson, A.; Hjort, M.; et al. Integration of biopolymer production with process water treatment at a sugar factory. New Biotechnol. 2014, 31, 308-323. [CrossRef] [PubMed]

9. Lim, S.-J.; Kim, B.J.; Jeong, C.-M.; Ahn, Y.H.; Chang, H.N. Anaerobic organic acid production of food waste in once-a-day feeding and drawing-off bioreactor. Bioresour. Technol. 2008, 99, 7866-7874. [CrossRef] [PubMed]

10. Komemoto, K.; Lim, Y.G.; Nagao, N.; Onoue, Y.; Niwa, C.; Toda, T. Effect of temperature on VFA's and biogas production in anaerobic solubilization of food waste. Waste Manag. 2009, 29, 2950-2955. [CrossRef]

11. Zhang, P.; Chen, Y.; Zhou, Q. Waste activated sludge hydrolysis and short-chain fatty acids accumulation under mesophilic and thermophilic conditions: Effect of pH. Water Res. 2009, 43, 3735-3742. [CrossRef]

12. Jiang, J.; Zhang, Y.; Li, K.; Wang, Q.; Gong, C.; Li, M. Volatile fatty acids production from food waste: Effects of $\mathrm{pH}$, temperature, and organic loading rate. Bioresour. Technol. 2013, 143, 525-530. [CrossRef]

13. Lee, W.S.; Chua, A.S.M.; Yeoh, H.K.; Ngoh, G.C. A review of the production and applications of waste-derived volatile fatty acids. Chem. Eng. J. 2014, 235, 83-99. [CrossRef]

14. Dahiya, S.; Sarkar, O.; Swamy, Y.V.; Mohan, S.V. Acidogenic fermentation of food waste for volatile fatty acid production with co-generation of biohydrogen. Bioresour. Technol. 2015, 182, 103-113. [CrossRef] [PubMed]

15. Erb, T.J.; Berg, I.A.; Brecht, V.; Müller, M.; Fuchs, G.; Alber, B.E. Synthesis of C5-dicarboxylic acids from C2-units involving crotonyl-CoA carboxylase/reductase: The ethylmalonyl-CoA pathway. Proc. Natl. Acad. Sci. USA 2007, 104, 10631-10636. [CrossRef] [PubMed]

16. Leroy, B.; De Meur, Q.; Moulin, C.; Wegria, G.; Wattiez, R. New insight into the photoheterotrophic growth of the isocytrate lyase-lacking purple bacterium Rhodospirillum rubrum on acetate. Microbiology 2015, 161, 1061-1072. [CrossRef] [PubMed]

17. Schneider, K.; Peyraud, R.; Kiefer, P.; Christen, P.; Delmotte, N.; Massou, S.; Portais, J.-C.; Vorholt, J.A. The ethylmalonyl-CoA pathway is used in place of the glyoxylate cycle by Methylobacterium extorquens AM1 during growth on acetate. J. Biol. Chem. 2012, 287, 757-766. [CrossRef]

18. Knight, M. The photometabolism of propionate by Rhodospirillum rubrum. Biochem. J. 1962, 84, 170. [CrossRef]

19. De Meur, Q.; Deutschbauer, A.; Koch, M.; Bayon-Vicente, G.; Cabecas Segura, P.; Wattiez, R.; Leroy, B. New perspectives on butyrate assimilation in Rhodospirillum rubrum S1H under photoheterotrophic conditions. BMC Microbiol. 2020, 20, 1-20. [CrossRef] [PubMed]

20. Cabecas Segura, P.; De Meur, Q.; Alloul, A.; Tanghe, A.; Vlaeminck, S.E.O.R.; Wouwer, V.; Wattiez, R.A.; Dewasme, L.; Leroy, B. Experimental Study and Dynamic Modeling of Preferential Photoassimilation in Volatile Fatty Acid Mixtures by Purple Non-Sulfur Bacteria; Laboratory of Proteomics and Microbiology, University of Mons: Mons, Belgium, 2021; to be Submitt.

21. Segers, L.; Verstraete, W. Conversion of organic acids to $\mathrm{H}_{2}$ by Rhodospirillaceae grown with glutamate or dinitrogen as nitrogen source. Biotechnol. Bioeng. 1983, 25, 2843-2853. [CrossRef]

22. Suhaimi, M.; Liessens, J.; Verstraete, W. $\mathrm{NH}^{+} / 4-\mathrm{N}$ assimilation by Rhodobacter capsulatus ATCC 23782 grown axenically and non-axenically in N and C rich media. J. Appl. Bacteriol. 1987, 62, 53-64. [CrossRef]

23. Snell, K.D.; Feng, F.; Zhong, L.; Martin, D.; Madison, L.L. YfcX enables medium-chain-length poly (3-hydroxyalkanoate) formation from fatty acids in recombinant Escherichia coli fadB strains. J. Bacteriol. 2002, 184, 5696-5705. [CrossRef]

24. Bradford, M.M. A rapid and sensitive method for the quantitation of microgram quantities of protein utilizing the principle of protein-dye binding. Anal. Biochem. 1976, 72, 248-254. [CrossRef]

25. Muhitch, M.J. Acetolactate synthase activity in developing maize (Zea mays L.) kernels. Plant Physiol. 1988, 86, 23-27. [CrossRef] [PubMed]

26. Westerfeld, W.W. A colorimetric determination of blood acetoin. J. Biol. Chem. 1945, 161, 495-502. [CrossRef]

27. Brock, M.; Maerker, C.; Schütz, A.; Völker, U.; Buckel, W. Oxidation of propionate to pyruvate in Escherichia coli: Involvement of methylcitrate dehydratase and aconitase. Eur. J. Biochem. 2002, 269, 6184-6194. [CrossRef]

28. Bayon-Vicente, G.; Marchand, E.; Ducrotois, J.; Dufrasne, F.E.; Hallez, R.; Wattiez, R.; Leroy, B. Analysis of the involvement of the isoleucine biosynthesis pathway in photoheterotrophic metabolism of Rhodospirillum rubrum. Front. Microbiol. 2021, $12,731976$. [CrossRef] 
29. Gordon, G.C.; McKinlay, J.B. Calvin cycle mutants of photoheterotrophic purple nonsulfur bacteria fail to grow due to an electron imbalance rather than toxic metabolite accumulation. J. Bacteriol. 2014, 196, 1231-1237. [CrossRef] [PubMed]

30. Evans, M.C.; Buchanan, B.B.; Arnon, D.I. A new ferredoxin-dependent carbon reduction cycle in a photosynthetic bacterium. Proc. Natl. Acad. Sci. USA 1966, 55, 928-934. [CrossRef]

31. Buchanan, B.B.; Arnon, D.I. A reverse KREBS cycle in photosynthesis: Consensus at last. Photosynth. Res. 1990, $24,47-53$. [CrossRef]

32. Bond-Watts, B.B.; Bellerose, R.J.; Chang, M.C.Y. Enzyme mechanism as a kinetic control element for designing synthetic biofuel pathways. Nat. Chem. Biol. 2011, 7, 222-227. [CrossRef] [PubMed]

33. Bayon-Vicente, G.; Wattiez, R.; Leroy, B. Global Proteomic Analysis Reveals High Light Intensity Adaptation Strategies and Polyhydroxyalkanoate Production in Rhodospirillum rubrum Cultivated With Acetate as Carbon Source. Front. Microbiol. 2020, 11, 1-17. [CrossRef] [PubMed]

34. Wang, D.; Zhang, Y.; Welch, E.; Li, J.; Roberts, G.P. Elimination of Rubisco alters the regulation of nitrogenase activity and increases hydrogen production in Rhodospirillum rubrum. Int. J. Hydrogen Energy 2010, 35, 7377-7385. [CrossRef] [PubMed]

35. Brämer, C.O.; Silva, L.F.; Gomez, J.G.C.; Priefert, H.; Steinbüchel, A. Identification of the 2-methylcitrate pathway involved in the catabolism of propionate in the polyhydroxyalkanoate-producing strain Burkholderia sacchari IPT101T and analysis of a mutant accumulating a copolyester with higher 3-hydroxyvalerate content. Appl. Environ. Microbiol. 2002, 68, 271-279. [CrossRef] [PubMed]

36. Silva, L.F.; Gomez, J.G.C.; Oliveira, M.S.; Torres, B.B. Propionic acid metabolism and poly-3-hydroxybutyrate-co-3-hydroxyvalerate (P3HB-co-3HV) production by Burkholderia sp. J. Biotechnol. 2000, 76, 165-174. [CrossRef]

37. Hädicke, O.; Grammel, H.; Klamt, S. Metabolic network modeling of redox balancing and biohydrogen production in purple nonsulfur bacteria. BMC Syst. Biol. 2011, 5, 150. [CrossRef]

38. Ormerod, J.G. The use of radioactive carbon dioxide in the measurement of carbon dioxide fixation in Rhodospirillum rubrum. Biochem. J. 1956, 64, 373. [CrossRef]

39. De Meur, Q.; Deutschbauer, A.; Koch, M.; Wattiez, R.; Leroy, B. Genetic plasticity and ethylmalonyl coenzyme A pathway during acetate assimilation in Rhodospirillum rubrum S1H under photoheterotrophic conditions. Appl. Environ. Microbiol. 2018, 84, e02038-17. [CrossRef]

40. Bayon-Vicente, G.; Zarbo, S.; Deutschbauer, A.; Wattiez, R.; Leroy, B. Photoheterotrophic Assimilation of Valerate and Associated Polyhydroxyalkanoate Production by Rhodospirillum rubrum. Appl. Environ. Microbiol. 2020, 86, e00901-20. [CrossRef]

41. McCully, A.L.; Onyeziri, M.C.; LaSarre, B.; Gliessman, J.R.; McKinlay, J.B. The reverse TCA cycle and reductive amino acid synthesis pathways contribute to electron balance in a Rhodospirillum rubrum Calvin cycle mutant. bioRxiv 2019, 614065. 